\title{
Tecnologías Digitales y Cambio Educativo. Una Aproximación Crítica
}

\section{Digital Technologies and Educational Change. A Critical Approach}

\author{
Manuel Area ${ }^{1, *}$, Jordi Adell ${ }^{2}$ \\ ${ }^{1}$ Universidad de La Laguna, España \\ ${ }^{2}$ Universidad Jaume I de Castelló, España
}

\section{DESCRIPTORES:}

Innovación educativa

Tecnología educativa

Educación digital

TIC

Escuela digital

\section{RESUMEN:}

Este artículo analiza y reflexiona sobre el impacto de las tecnologías digitales en la transformación pedagógica del sistema escolar desde posicionamientos de la tecnología educativa crítica. Para ello, se comienza identificando los rasgos o efectos más destacables de lo que debiera suponer la disrupción pedagógica de la tecnología sobre la escuela. A partir de los mismos, se abordan y analizan cuatro temáticas o tópicos actualmente relevantes como son a) la Covid19 y el cierre de los centros escolares: el inesperado impulso de la enseñanza digital; b) la integración pedagógica y organizativa de las TIC en los centros escolares; c) la metamorfosis del material didáctico. De lo analógico a lo digital; y d) la competencia digital docente. El análisis se realiza entre las taxonomías de microhabilidades y los enfoques holísticos. Finaliza el artículo concluyendo que aún no se ha producido una disrupción digital de la institución escolar, pero que se avanza en dicha dirección. La misma no sólo debe innovar los métodos y estrategias de enseñanza, sino también el sentido, las metas, la organización académica, las funciones profesionales de los docentes, y en última instancia, la funcionalidad de la escuela en el contexto de la sociedad digital.
\end{abstract}

\section{KEYWORDS:}

Educational innovation

Educational technology

Digital education

ICT

Digital school

\section{ABSTRACT:}

This article analyzes and reflects on the impact of digital technologies on the pedagogical transformation of the school system from the standpoint of critical educational technology. To this end, it begins by identifying the most salient features or effects of what the pedagogical disruption of technology on the school should entail. Based on these, four currently relevant themes or topics are addressed and analyzed, such as a) Covid 19 and the closure of schools: the unexpected impulse of digital instruction; b) The pedagogical and organizational integration of ICT in schools; c) The metamorphosis of didactic materials. The analysis is made from analog to digital resources; and d) digital teaching competence. Between micro-skills taxonomies and holistic approaches. The article ends by concluding that there has not yet been a digital disruption of the school institution, but that progress is being made in that direction. It must not only innovate teaching methods and strategies, but also the meaning, goals, academic organization, professional functions of teachers, and ultimately, the functionality of the school in the context of the digital society.

\section{CÓMO CITAR:}

Area, M. y Adell, J. (2021). Tecnologías digitales y cambio educativo. Una aproximación crítica. REICE. Revista Iberoamericana sobre Calidad, Eficacia y Cambio en Educación, 19(4), 83-96. https://doi.org/10.15366/reice2021.19.4.005

*Contacto: manarea@ull.edu.es

ISSN: $1696-4713$

revistas.uam.es/reice 


\section{Introducción}

El primer cuarto del siglo XXI probablemente será recordado como la época donde la tecnología digital invadió y se apropió de todas las esperas y ámbitos de la organización social, económica y cultural de la sociedad humana. Como señaló a finales del siglo XX Postman, este estadio evolutivo de la civilización humana, es el triunfo de Tecnópolis (Postman, 1994). En apenas dos décadas la telefonía móvil, el big data, la inteligencia artificial, la robótica, la realidad virtual, entre otras muchas tecnologías digitales, han pasado de ser prototipos experimentales en laboratorios o centros de investigación de Silicon Valley a ser tecnologías extendidas e incorporadas al funcionamiento de las organizaciones empresariales, del ocio, de los servicios de administración y gestión gubernamental, así como de nuestros hogares.

La revolución digital, además de la aparición y expansión de nuevos dispositivos, servicios telemáticos y máquinas inteligentes, representa una profunda alteración de las formas de relacionarnos, de socializarnos, de producir, consumir, difundir y acceder a la cultura y el conocimiento y, en consecuencia, una metamorfosis de nuestra existencia como humanos (Cobo, 2019). Ante este nuevo estadio o fase de la civilización cabe plantearse ¿qué papel ha de jugar la educación en un contexto social, cultural y económico gestionado y controlado por redes de máquinas interconectadas e inteligentes? ¿qué significa y en qué consiste ser ciudadanos plenos, cultos y autónomos que viven en una sociedad totalmente digitalizada? ¿Cómo debe reformularse el curriculum y el sistema escolar para dar respuesta adecuada a estas necesidades? En pocas palabras ¿cómo educarse en la era digital? (Pérez Gómez, 2012).

En este artículo pretendemos analizar y reflexionar sobre algunos de los problemas actuales en torno al proceso de integración y transformación digital del sistema escolar desde un enfoque o perspectiva que quiere superar una visión tecnocentrista o instrumentalista del impacto de la tecnología sobre la educación (Adell, 2018) y que mira más allá de los artefactos tecnológicos (Area et al., 2020).

En el panorama internacional existen distintos trabajos que han realizado notorias revisiones sobre lo publicado en el ámbito de las aplicaciones de las tecnologías a la educación y los efectos de las mismas. Entre las mismas podemos destacar el artículo de Hsu y otros (2013) en el que analizaron más de tres mil artículos publicados en el Social Science Citation Index, la revisión realizada por West y Borup (2014), así como el trabajo de Bond y otros (2018) donde exploraron casi dos mil títulos y resúmenes de artículos de investigación, publicados en el British Journal of Educational Technology (BJET) en el periodo de 1970 a 2018. De forma similar Natividad y otros (2018) examinaron el estado de la investigación en el campo de la Tecnología Educativa a través de la revisión de lo publicado en las diez revistas internacionales más destacadas. En el contexto español contamos con trabajos que, sin ser revisiones bibliográficas, abordan una aproximación al estado de la investigación en torno a la tecnología en la educación (Cabero, 2016; Gros, 2016; Marín et al., 2017; Castañeda, Salinas y Adell, 2020).

Nuestra mirada o enfoque, a diferencia de los trabajos referidos elaborados con una racionalidad cuantitativa y bibliométrica, está más próximo al ensayo interpretativo sobre la tecnología en la enseñanza donde se entremezcla el análisis estrictamente didáctico de sus efectos con otras variables de naturaleza organizativa, política y sociocultural. Responde a la tradición representada por lo que pudiera denominarse, quizás de un modo impreciso, como una tecnología educativa crítica (Burbules y Callister, 2001; Cuban, 1986; Nichols y Allen-Brown, 1996; Selwyn, 2011, 2014; entre otros).

\section{Tecnologías en el sistema escolar ¿cuándo son disruptivas?}

Somos conscientes de que, aunque históricamente algunas tecnologías han sido disruptivas en ocasiones no siempre ocurre así, sino que se acomodan, se domestican y pierden su potencial transformador. Esto ocurre porque los efectos socioculturales de una tecnología no dependen solamente de sus propias características artefactuales, sino también y, sobre todo, de otras condiciones de naturaleza contextual como son las creencias y prácticas de sus usuarios, de los intereses económicos o políticos que las rodean, o de la utilidad práctica de las mismas en la resolución de problemas percibidos como relevantes.

En el ámbito escolar llevamos varias décadas intentado incorporar las tecnologías digitales al sistema escolar. A pesar de los avances realizados en la dotación de infraestructuras y recursos telemáticos, en la creación de materiales didácticos en línea, de oferta de servicios en la red, en la formación y competencia 
digital del profesorado, ... en numerosas ocasiones la presencia de las TIC en los centros y aulas no ha representado más que un mero cambio epidérmico o cosmético de las prácticas de enseñanza y aprendizaje que allí ocurren.

El ejemplo más evidente es la utilización de los cañones de proyección y las pizarras digitales que las acompañan que, en ocasiones, han servido para reforzar el papel de las lecciones o clases magistrales impartidas por el docente. En otras ocasiones, la digitalización de las tareas de los estudiantes no consiste en que éstos empleen recursos online interactivos o multimedia, sino simplemente en el escaneo o digitalización de sus cuadernos manuscritos de papel para ser enviados a su docente a través de Internet. Otro ejemplo, de cómo la tecnología no altera radicalmente lo que ya existente es la conversión de los libros de texto en soporte impreso en ebooks escolares que prácticamente son los mismos ofertados en papel, pero para ser leídos en pantallas. En otras palabras, para seguir haciendo lo mismo que antes no merece la pena el coste económico ni el esfuerzo organizativo de la incorporación de las tecnologías a las escuelas.

La pregunta en consecuencia es ¿cuándo podemos considerar que las tecnologías son disruptivas pedagógicamente e impulsan el cambio educativo? (Cabrol y Severin, 2010; Christensen et al., 2008; Magana, 2017). En otras palabras ¿cómo se manifiesta o qué fenómenos evidencian que las mismas alteran de forma decisiva la enseñanza tradicional? ¿Estamos asistiendo a la extensión de pedagogías emergentes (Adell y Castañeda, 2012, 2015)?

A continuación, vamos a plantear y hacer revisión del estado en el que se encuentran algunas de las líneas o temáticas de investigación más destacadas sobre el ámbito de la Tecnología Educativa en lo que se refiere a las políticas y prácticas sobre cómo las mismas están impulsando el cambio educativo en la realidad de los centros y aulas del sistema escolar. Para ello hemos organizado este análisis de las políticas y prácticas en torno a cuatro temáticas o tópicos. Los mismos responden, por una parte, a algunas de las temáticas sobre las que más se está publicado en los últimos años en las revistas del campo y, por otra, a un proceso de debate y consenso realizado por los autores en calidad de expertos del campo de la tecnología educativa en el contexto español. Estos tópicos son los siguientes:

- La Covid 19 y el cierre de los centros escolares: el inesperado impulso de la enseñanza digital

- La integración pedagógica y organizativa de las TIC en los centros escolares

- La metamorfosis del material didáctico. De lo analógico a lo digital

- La competencia digital docente. Entre las taxonomías de microhabilidades y los enfoques holísticos.

\section{La Covid 19 y el cierre de los centros escolares: El inesperado impulso de la enseñanza digital}

Sobre el impacto escolar del confinamiento provocado por la pandemia Covid19 se han publicado una enorme cantidad de estudios, artículos y ensayos que han explorado las experiencias, soluciones y prácticas desarrolladas. En este sentido distintos organismos internacionales como la OCDE (OECD and Harvard Graduate School of Education, 2020; Reimers y Schleicher, 2020a, 2020b) o la UNESCO (2020a, $2020 b, 2020 c$ ) han intentado extraer lecciones de lo ocurrido y proponer orientaciones y guías para el tiempo pospandémico.

En el contexto mundial, pero en el caso español en particular, la tecnología fue la respuesta de urgencia. Inesperadamente, no solo en el ámbito educativo, sino en todos los sectores socioeconómicos, las redes digitales se convirtieron en las protagonistas para continuar realizando las actividades de compra, de entretenimiento, de trabajo, de comunicación, entre otras. Durante el confinamiento emergieron con fuerza inusitada el teletrabajo, las telecompras, el consumo digital de los productos culturales, la interacción e intercambios comunicativos con familiares y amistades a través de las teleconferencias. Lo tecnológico o virtual de convirtió en el núcleo central de la existencia cotidiana. Ello, inevitablemente, trajo consigo el crecimiento exponencial de los servicios digitales y que las empresas tecnológicas, de telecomunicacio- 
nes y del ciberespacio fueran las grandes beneficiadas de la pandemia frente a otros numerosos sectores de la economía de lo tangible que han entrado en crisis.

El confinamiento generó la inmediata desaparición de la enseñanza presencial para ser sustituida de forma abrupta y apresurada por la mediación educativa a través de tecnologías tanto síncronas (como las videollamadas en tiempo real) como asíncronas (los entornos virtuales). Por esta razón todas las administraciones educativas internacionales, nacionales y autonómicas organizaron e impulsaron un modelo improvisado o de urgencia para la enseñanza digital desarrollado desde el hogar por el profesorado y el alumnado. Pudiéramos señalar que las medidas y decisiones que se tomaron, entre otras, fueron:

La utilización de los sistemas de videoconferencias o reuniones telemáticas para la celebración de las reuniones del claustro de profesorado cara coordinar actuaciones de ciclo, nivel o materia, para acordar medidas académicas o regular las evaluaciones.

La utilización de estos sistemas de videollamadas o telereuniones por parte del profesorado con su alumnado para intentar continuar, en la medida de lo posible, la acción docente estableciendo tareas y actividades, supervisando y guiando las mismas, explicando contenidos, o realizando pruebas evaluativas.

La potenciación de los entornos o plataformas LMS (Learning Management System) tipo Moodle, Blackboard y similares para que el profesorado generara espacios o aulas virtuales para el seguimiento del trabajo académico del alumnado (ofrecer materiales de estudio, entrega de tareas escolares, mantener comunicación asíncrona, ...).

La creación y difusión de portales web que recopilaban y clasificaban, a modo de bibliotecas en línea, aquellos recursos y materiales didácticos digitales para que el profesorado los seleccione y utiliza con su alumnado.

La selección y difusión de apps, software y programas informáticos que les permitieran tanto al profesorado como alumnado la creación de contenidos digitales de distinta naturaleza como videos, infografías, animaciones, podcast, etc.

La creación y distribución a través de canales de televisión públicos, tanto a nivel nacional como autonómico, de programas emitidos en horario escolar sobre los contenidos curriculares destinados al alumnado.

Acuerdos con compañías y corporaciones de telecomunicaciones y de servicios digitales que, bien de forma gratuita o a precio reducido, permitieran el uso de sus productos con fines educativos de forma temporal.

Organización de cursos, webinar y otras actividades online de formación al profesorado sobre el uso de las TIC.

La pandemia, y en particular el confinamiento, pusieron en evidencia fenómenos que ya existían previamente, pero que se agudizaron con esta nueva situación que, entre otras, fueron:

La ausencia de la cualificación adecuada del profesorado en el ámbito o campo profesional de la tecnología educativa. Esto significó que una parte importante y notoria de los docentes no solo no disponían de suficientes competencias digitales, sino que también carecían de los conocimientos y destrezas para planificar, desarrollar y evaluar situaciones educativas gestionadas a través del ciberespacio tanto de forma síncrona como asíncrona. A pesar de ello, hay que reconocer el enorme y valioso esfuerzo realizado por la inmensa mayoría del profesorado para hacer frente a todos estos obstáculos y comprometerse con la continuidad de su docencia mediante estas modalidades de enseñanza a distancia en línea.

Las diferencias o brechas digitales entre el alumnado por su origen y situación socioeconómica familiar. El confinamiento provocó que aquel alumnado que carecía de tecnología o que disponía de conexión limitada y de baja calidad a Internet tuviera grandes dificultades para continuar sus estudios desde el hogar frente al alumnado de ambientes familiares y socioeconómicos favorecidos. Esto hizo visible, una vez más, las diferencias entre el alumnado de la red pública y la privada de la escolaridad. En otras palabras, la Covid 19 ha incrementado las desigualdades entre un alumnado y otro (Alto Comisionado para la Lucha contra la Pobreza Infantil, 2020; Cabrera et al., 2020).

El desarrollo creciente del sector industrial privado que se conoce como Edutech. Aunque viene gestándose desde años atrás, es indudable que la Covid19 ha impulsado y consolidado este mercado sobre las 
tecnologías aplicadas a la educación (Willianson y Hogan,2020). Las grandes corporaciones tecnológicas (Amazon, Google, Microsoft, ...) han descubierto el enorme potencial que existe en el ámbito de la educación y compiten con aquellas que tradicionalmente se dedicaron a la creación y comercialización de productos y materiales educativos. Campos como el elearning, la gestión y administración digital de los servicios de los centros, la creación y mantenimiento de plataformas de contenidos educativos, la dotación y supervisión de infraestructuras, artefactos y servicios tecnológicos en la red, las analíticas de datos, la inteligencia artificial aplicada a la educación, la robótica educativa, entre otras muchas, son productos de interés que probablemente incrementarán su oferta en los próximos años desde el ámbito de la industria Edutech.

\section{La integración organizativa y pedagógica de las TIC en los centros escolares}

Uno de los problemas o temática más investigada en los últimos años es el referido a la integración pedagógica y organizativa de la tecnología digital en los centros y aulas escolares (BECTA, 2004; Ertmer, 1999; European Schoolnet, 2006; Hew y Brush, 2007; Pelgrum, 2001). Estos trabajos, entre otros, encontraron que existen una serie de condiciones que suelen ser repetirse independientemente del contexto y que parecen estar vinculadas con el uso pedagógico e innovador de las tecnologías. Estos factores se refieren al grado de habilidades o competencia digital del profesorado en la utilización del hardware como del software; la confianza, expectativas y actitudes de los mismos hacia el uso de las TIC en su enseñanza; la cantidad de las infraestructuras y recursos informáticos que tiene a su disposición en los centros y aulas; la calidad de la conectividad de los mismos a la red; las experiencias previas en el desarrollo de proyectos basados en la utilización de las TIC; el clima innovador y cultura organizativa del centro escolar; los servicios de apoyo externo facilitadores de la utilización de las tecnologías; así como el grado de flexibilidad o de autonomía curricular de los centros. Según la combinación e interrelación de los mismos que se produzca en un centro impulsarán o neutralizarán la utilización de las tecnologías con fines educativos.

Por ello se han identificado distintos estadios o niveles de integración pedagógica de las tecnologías en los centros educativos en cuanto organizaciones (Valverde, 2015). Esta línea o enfoque de estudios ha establecido un continuum de grados o etapas que suelen desarrollar los centros escolares en su proceso de apropiación y uso de las tecnologías (Kampylis et al., 2015; Lugo y Kelly, 2011; Severin, 2011; Severin et al., 2012;). De este modo, han identificado estadios iniciales, medios y avanzados en el proceso de integración y digitalización de las organizaciones educativas (Area, 2015; Area et al., 2020). De forma similar el Departamento de Educación, Universidades e Investigación del Gobierno Vasco (2008) propuso un modelo de madurez tecnológica del centro educativo similar a este planteamiento.

La presencia y disponibilidad de las tecnologías digitales en los centros y aulas se ha normalizado, es decir, ha dejado de ser un hecho extraordinario. Actualmente los ordenadores, los cañones de proyección o las tabletas digitales son un elemento más y cotidiano del paisaje escolar. Hace ya dos décadas atrás Gros (2000) se refirió a este fenómeno como la invisibilización escolar de los ordenadores.

Desde entonces hasta hoy se han producido muchas transformaciones radicales y rupturistas, más fuera que dentro de la escuela, pero que han empujado a que en el interior de la arquitectura de los espacios educativos las diversas tecnologías ya convivan en la práctica diaria de los docentes y los estudiantes. Uno de los hechos más evidentes de este proceso de normalización escolar de la tecnología es la lenta, pero constante desaparición de las conocidas como "salas de ordenadores" o "aulas de informática" de los colegios para descentralizarse y ubicarse en las aulas ordinarias de clase.

Este hecho puramente técnico-organizativo junto con el incremento de la conectividad inalámbrica o wifi está teniendo consecuencias pedagógicas muy relevantes. La primera de ellas es que el alumnado no tiene que salir de su clase y desplazarse físicamente a otro lugar del centro para desarrollar actividades con los ordenadores. La segunda consecuencia es que al profesorado se le simplifica las tareas de planificación, organización y gestión de situaciones didácticas de uso de las TIC: no tiene que solicitar permisos con anterioridad, ni tiene que buscar un hueco en los horarios de disponibilidad de dichas salas. 
Otra evidencia de la normalización se refiere a que el alumnado, en su mayor parte, dispone de tecnologías propias o personales bien de un ordenador portátil, de un smartphone o de una tableta digital. La reducción del tamaño de los artefactos tecnológicos y de su coste económico junto con el aumento de su potencia de procesamiento está facilitando la facilidad en su transporte y movilidad y que sea accesible para mucho alumnado.

Internacionalmente, hace ya una década, se desarrollaron programas y políticas educativas que tenían precisamente la finalidad de que cada estudiante dispusiera de su propia tecnología. Fue conocido como el modelo 1 a 1 que tuvo una importante difusión en Latinoamérica (Area, 2011; Valiente, 2011). En nuestro país fue impulsado por el programa Escuela 2.0. Este programa fue efímero como consecuencia de la profunda crisis económica de la época que obligó a recortes de las inversiones públicas, así como por el cambio del partido de gobierno. Han pasado casi diez años desde entonces, pero el eco y huella de aquel Programa sigue en cierta medida vigente en el sentido de que cada alumno comienza a disponer de su propia tecnología para las tareas escolares.

Al llenarse los hogares tanto del profesorado como del alumnado con tecnologías y servicios digitales diversos (teléfonos inteligentes, televisión digital, tabletas, fibra óptica, redes sociales, plataformas audiovisuales, ordenadores portátiles o de sobremesa, ...) los agentes educativos no sólo desarrollaron las habilidades de uso de los mismos, sino que interiorizaron la necesidad de utilizarlos de modo constante en su vida cotidiana para múltiples actividades como obtener información, divertirse, comprar, relacionarse con otros, trabajar, realizar trámites administrativos, gestionar sus finanzas o trámites bancarios, jugar, escuchar música, consumir productos audiovisuales, ..., entre otras muchas acciones habituales de la existencia humana.

Casi sin querer el profesorado y el alumnado, al margen de lo escolar, comenzó a dotarse de su propia tecnología, a desarrollar los conocimientos y habilidades técnicas de su uso y a concienciarse de su necesidad de las mismas para desenvolverse como ciudadano de la sociedad del presente.

Los centros educativos privados apostaron decididamente por invertir e incorporar las nuevas tecnologías a sus prácticas de enseñanza no sólo como reclamo para la formación del alumnado en las nuevas competencias para la sociedad del siglo XXI, sino también para modernizar sus métodos didácticos apostando por metodologías de aprendizaje activo que están de moda como el flipped classroom, el aprendizaje por proyectos, la gamificación o la robótica educativa, entre otros.

Una última consideración: si de forma paralela no se desarrollan políticas de inversiones suficientes en los centros escolares públicos este hecho podrá provocar el incremento de la brecha digital, a la que ya aludimos anteriormente, entre el alumnado de una red escolar y otra como ya ha evidenciado la experiencia del confinamiento por la pandemia.

\section{La metamorfosis del material didáctico. De lo analógico a lo digital}

Otro tema relevante se refiere al proceso lento, pero continuado de transformación digital que está ocurriendo con relación a los materiales o recursos didácticos. Es un proceso, aunque con ciertas variantes propias de la idiosincrasia escolar, similar al que acontece con el resto de las industrias culturales donde los medios analógicos están desapareciendo para ser sustituidos por objetos digitales distribuidos en línea (Area, 2017).

La institución escolar, en su origen decimonónico, es deudora de la hegemonía de la cultura impresa de su época. La alfabetización en las competencias de lectura y escritura fue una de sus señas de identidad y justificación de la escuela durante el S. XIX y gran parte del XX. La historia de la institución escolar está íntimamente ligada a esa meta y función social. Por esta razón, entre otras, los libros y demás instrumentos de la cultura impresa han sido el eje o patrón dominante de la actividad académica en las aulas. El libro de texto, al menos en estas últimas décadas, fue el medio o material didáctico que ha monopolizado los procesos de enseñanza-aprendizaje escolares. Existe bastante bibliografía en español al respecto y no reiteraremos lo ahí aportado (Gimeno, 2011; Martínez Bonafé, 2010).

Lo que queremos evidenciar es que la llegada y expansión de las tecnologías en las escuelas está provocando el cuestionamiento y la ruptura con los modelos pedagógicos que representa el texto como canon de la práctica curricular. En esta ocasión la tecnología es disruptiva porque desplaza a los libros de textos en soporte 
de papel y porque altera los métodos tradicionales de enseñanza basados en la utilización continuada del texto escolar.

En otros trabajos ya hemos explorado el impacto de la cultura digital sobre la transformación del material didáctico y a ellos remitimos al lector (Area, 2017, 2020). Grosso modo, podríamos sintetizar que los cambios o mutaciones tanto técnicas como pedagógicas que acompañan e impulsan esta nueva generación de materiales didácticos en formato digital y que son accesibles a través del ciberespacio, entre otros, son los siguientes:

- La deslocalización del conocimiento que pasa de estar encerrado en un soporte físico que lo empaqueta a un territorio virtualizado sin límites definidos.

- La ruptura con el modelo enciclopedista en la organización y presentación didáctica del conocimiento a través de materias estancas para abrirse a combinaciones de distintas disciplinas ofrecidas a través de proyectos de trabajo o temáticas transdisciplinares.

- La apertura a formatos expresivos más allá de las codificaciones alfabéticas del conocimiento incorporando los lenguajes iconográficos, audiovisuales, sonoros y representaciones tridimensionales.

- La llegada de nuevas narrativas y experiencias cognitivas consecuencia de los hirpertextos, la interactividad, el transmedia, la realidad aumentada y los escenarios de realidad virtual.

- La automatización del procesamiento de datos que mediante la inteligencia artificial está evolucionando a que el material didáctico deje de ser estandarizado y homogéneo para todo el alumnado para personalizar su contenido, interface y tareas en función de los rasgos y comportamientos particulares de cada estudiante.

- El empoderamiento que ofrece al profesorado y a los estudiantes como creadores de contenidos y objetos digitales que pueden ser elaboradores, difundidos y compartidos sin gran coste económico ni requiriendo un conocimiento técnico experto.

La transformación digital del material didáctico también es disruptiva con el modelo de negocio de las industrias editoriales que editan estos recursos de enseñanza. La principal asociación del ramo ANELE (Asociación Nacional de Editores de Libros y material de Enseñanza) en el informe del año 2010 señalaba que "la venta de libros de texto digitales es todavía irrelevante... representa el $0.04 \%$ de la cifra de negocio del libro educativo". Curiosamente diez años después en el último informe de 2020 indica que se siguen publicando un número similar de libros de texto en papel. Por el contrario, la edición de libros digitales ha incrementado de forma espectacular su cantidad de títulos (más de 18.000). Sin embargo, y este es un dato muy llamativo, su peso o monto económico sigue siendo escaso ya que solamente representa menos del $4 \%$ con relación al total de los ingresos generados por los libros de texto en papel.

Es desconcertante que en los tiempos actuales de omnipresencia de la tecnología digital en todas las esferas de nuestra sociedad todavía el material impreso siga siendo dominante. Suponemos que este es una de las señas diferenciales e idiosincráticas del sistema escolar que, en algunos aspectos, todavía es refractario a las transformaciones profundas que impulsa la revolución informática y de telecomunicaciones. Pero cabe otra lectura.

Si analizamos lo ocurrido en otros sectores de la industria y consumo cultural como la prensa escrita, la industria musical o la audiovisual podríamos sugerir que la transformación digital del sistema de producción y consumo del material didáctico lleva un retraso de diez años. Aquellas tuvieron que reinventarse para responder a las nuevas pautas de acceso y consumo digital, a las formas de elaboración y distribución de los contenidos o productos culturales, a los modelos de negocio de la economía digital, a los formatos multimedia y transmedia.

En definitiva, el campo de los materiales didácticos será a corto y medio plazo donde se harán visibles las transformaciones del impacto de lo digital sobre la educación escolar. Más pronto que tarde las mochilas que contienen libros de texto en papel para cada asignatura y que cargan los estudiantes cuando acuden desde su hogar a los colegios e institutos empiezan a ser sustituidas por una computadora portátil o una 
tableta digital que le permite a ese alumnado conectarse a la red para trabajar con plataformas o entornos online con multitud y variedad de recursos educativos desde cualquier lugar y en cualquier momento.

\section{La competencia digital docente. Entre las taxonomías de microhabilidades y los enfoques holísticos}

Este tema es uno los que está de moda actualmente en el panorama educativo. Al respecto existe una amplia y variada bibliografía sobre este concepto desde hace varios años tanto a nivel nacional como internacional. Sobre el mismo existe consenso que la competencia digital del profesorado es imprescindible si se pretende que el sistema educativo innove sus metodologías de enseñanza mediante el uso de las TIC para que el alumnado obtenga experiencias valiosas de aprendizaje. Hace años Cabero y otros (1998) señalaron que la formación y competencia del profesorado representaba la piedra angular para el uso de las distintas tecnologías en la enseñanza. De modo similar en la misma época (Yanes y Area, 1998) indicaban que la formación del profesorado como usuario de las nuevas tecnologías de la información en las escuelas no era sólo un problema técnico, sino también político e ideológico: En concreto afirmaban:

La formación de los docentes para el uso pedagógico de las nuevas tecnologías de la cultura digital tiene que vincularse forzosamente con la discusión de cuestiones relativas a los presupuestos ideológicos y políticos subyacentes en el cambio educativo que supuestamente quiere ser promovido con la incorporación de las nuevas tecnologías a la escolaridad; con la configuración de la cultura que debe transmitir la escuela en un entorno social en que las tecnologías digitales de la información están omnipresentes, con el debate sobre el nuevo papel que debe jugar el profesorado como agente socializador, con el sentido y utilidad de la presencia pedagógica de las nuevas tecnologías en las escuelas. (Yanes y Area, 1998, p. 26)

Sin docentes cualificados tanto digital como pedagógicamente no podrán implementarse proyectos educativos valiosos e innovadores con las tecnologías (Fuentes et al., 2019). Al respecto existen diversos informes con propuestas de dimensiones, estándares e indicadores de la competencia docente para la utilización de las tecnologías en la enseñanza (INTEF, 2017; ISTE, 2017; Redecker y Punie, 2017; UNESCO 2008a; 2018b).

Este último es el que ha inspirado el recientemente aprobado Marco de referencia de la competencia digital docente consensuado entre el Ministerio de Educación y todas las Consejerías de Educación de los gobiernos autónomos de nuestro país lo cual es un hito relevante y que lo convierte en un instrumento sólido y referencial para elaborar planes y políticas tanto de formación inicial como continua del profesorado con relación a su cualificación en el ámbito de la tecnología educativa. Brevemente hemos de indicar que este marco común está estructurado, siguiendo el modelo de competencia digital de la ciudadanía o DigComp 2.0 (Vuorikari et al., 2016) del centro JRC de la Unión Europea, por cinco grandes áreas: Área 1. Información y alfabetización informacional; Área 2. Comunicación y colaboración; Área 3. Creación de contenidos digitales; Área 4. Seguridad, y Área 5. Resolución de problemas.

Cada una de estas áreas se subdividen en dimensiones competenciales que están descritas en niveles o grados de dominio de la misma (básico, medio y avanzado) los cuales, a su vez, se organizan en otros seis niveles que expresan las acciones concretas que evidencian el dominio de la competencia. Supuestamente este marco intenta objetivar, agrupar y ordenar en términos de conducta las habilidades específicas del saber hacer docente cuando utiliza las tecnologías. En otras palabras, se ha pretendido crear un instrumento, a modo de taxonomía o clasificación, que visibilice el grado de apropiación y desarrollo competencial digital docente en términos microconductuales. De este modo, cualquier profesor o profesora podrá autoevaluarse o ser sometido a evaluación externa cara a diagnosticar o identificar el grado de dominio de la competencia digital.

Existen sin embargo otras aproximaciones conceptuales y analíticas a esta competencia que cuestionan este enfoque conductual y que persiguen describirla con un enfoque holístico, más comprensivo y funcional (Castañeda et al., 2018; Esteve et al., 2018). Estos autores critican este tipo de clasificaciones de la competencia digital docente por las siguientes razones: 
En primer lugar, que no parten explícitamente de un modelo de acción docente y de manera implícita reducen la función docente al trabajo en el aula, evitando aspectos como el compromiso social y político o el papel de la escuela en el desarrollo comunitario. En segundo lugar, que parten de una visión taxonómica del concepto de competencia, ignorando la complejidad de los diferentes contextos en los que dicha competencia se pone en acción y se desarrolla y en su papel en la construcción de la identidad del docente, algo que enfoques sociomateriales y holísticos sí incluyen. En tercer lugar, que se fundamentan de manera contradictoria en una visión instrumentalista de la tecnología como herramienta neutra en valores y, por el contrario, en una concepción determinista de la relación entre tecnología y sociedad. (Castañeda et al., 2018, p. 1)

Como alternativa proponen un modelo holístico y sistémico de dicha competencia en términos de funciones más amplias vinculadas con sus tareas profesionales (Figura 1)

Figura 1.

Modelo holístico de las competencias digitales

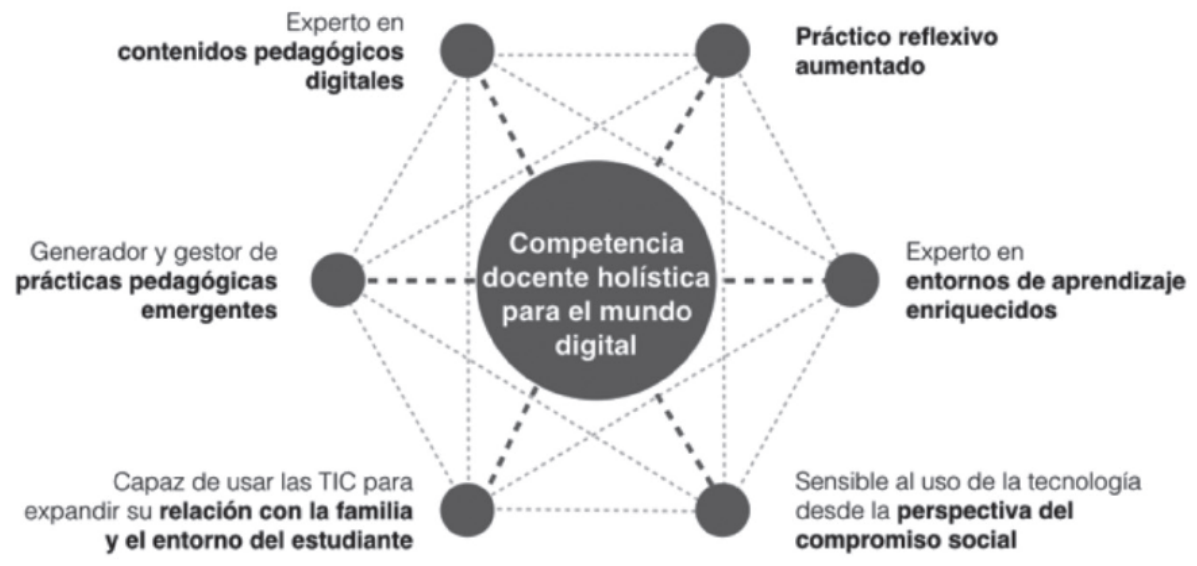

Nota. Recuperado de Castañeda y otros (2018).

Por otra parte, otra propuesta que también podría considerarse perteneciente a un enfoque sistémico sobre la competencia digital docente es el conocido internacionalmente como el modelo TPACK (Technological Pedagogical Content Knwoledge) formulado hace ya más de una década por Mishra y Koehler (2006) y reformulado en Harris y otros (2009). El mismo intenta explicar las acciones competenciales del profesorado en el cruce o simbiosis de tres ámbitos o dominios de conocimiento: el tecnológico, el pedagógico y el disciplinar (Figura 2).

Figura 2

Modelo TPACK

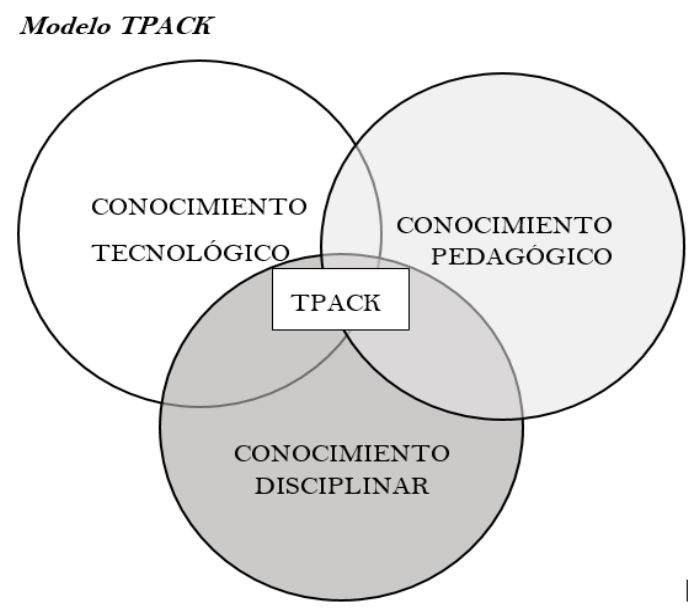


Quisiéramos también referirnos que a pesar de las importantes inversiones económicas realizadas en los últimos años con relación a la formación del profesorado para el uso de las tecnologías digitales tanto desde el gobierno central a través del INTEF (Instituto Nacional de Tecnologías Educativas y Formación del Profesorado) mediante estrategias como los MOOC, cursos online, cursos de verano, pero sobre todo desde las Consejerías de Educación autonómicas y en particular a través de los centros de profesorado o de formación en centros, sigue existiendo una percepción en el profesorado caracterizada por una débil e insuficiente cualificación para integrar y usar pedagógicamente las tecnologías en su práctica educativa.

De forma similar Escudero y otros (2018) en un estudio realizado sobre la formación en TIC ofertada desde los gobiernos autónomos encontraron que existe una alta demanda por el profesorado de este tipo de contenido formativo, que la modalidad más habitual es de cursos breves focalizados sobre herramientas digitales que son realizados de modo individual sin que sean contextualizados y aplicados a las prácticas particulares de los docentes, y que también es frecuente la formación autodidacta online. Prima un modelo formativo en TIC basado en el aprendizaje individual, descontextualizado e instrumentalista señalando que sigue existiendo una brecha entre lo declarado como deseable con relación a la integración escolar de las tecnologías y su materialización o traslado a la realidad de los centros y aulas. Estos autores, al respecto, concluyen que:

\begin{abstract}
Por más que se formulen competencias digitales docentes y organizativas, si la formación docente no trata debidamente las cuestiones curriculares y pedagógicas relevantes y sustantivas, la calidad de la educación y la integración de lo digital dentro de ella pueden quedar en entredicho... Por más que las políticas educativas conviertan las TIC en temas "estelares" y se dediquen más medios a la formación docente, el devenir de la deseable integración de las mismas será incierto, azaroso, aislado y no sistémico. (pp. 75-76)
\end{abstract}

Finalmente hemos de aludir a la limitada formación inicial que desde las Facultades de Educación se presta en los títulos de grado (Maestro de Educación Infantil, Maestro de Educación Primaria) y de posgrado (Profesorado de Educación Secundaria) al ámbito de la cualificación en Tecnología Educativa del futuro profesorado. Todavía en algunas universidades esta formación sigue siendo opcional o tiene una mínima presencia en los actuales planes de estudio. Ello nos obliga a demandar una profunda revisión de las titulaciones universitarias de formación del profesorado que incorpore decididamente una cualificación tanto teórica como práctica sobre este ámbito (García-Zabaleta et al., 2021; Lores et al., 2019; Sancho et al., 2015).

\title{
7. A modo de conclusiones
}

Una primera conclusión es que todavía no se ha producido una disrupción digital del sistema educativo, pero las evidencias empiezan a sugerir que vamos camino de ello. Nos encontramos en un cambio de época histórica caracterizado por profundas transformaciones socioeconómicas y culturales impulsadas por la omnipresencia de la tecnología digital. Esta mutación o transformación está provocando que todas las instituciones educativas, sean de ámbito escolar, de formación ocupacional, de centros de adultos, de educación no formal o de enseñanza universitaria están experimentado un proceso de transformación lento, pero gradual que consiste no sólo en la introducción de las tecnologías, sino un replanteamiento de mayor profundidad y alcance que va más allá de las innovaciones superficiales de la epidermis pedagógica, de las técnicas didácticas o de las prácticas de aula utilizando diversas y variadas tecnologías. Nos referimos a la disrupción transformadora no sólo los métodos y estrategias de enseñanza, sino del sentido, la finalidad, las metas, la organización tanto espacial como temporal académicas, las funciones profesionales de los docentes, y en última instancia, del valor y funcionalidad de la escuela en el contexto de la sociedad digital.

La revolución digital está provocando nuevos desafíos para la escuela del siglo XXI para educar a los niños y niñas, a los adolescentes y jóvenes como ciudadanos educados, críticos y competentes para desarrollarse con éxito en la sociedad digital. Por ello, las tecnologías representan una gran oportunidad para el cambio educativo ya que permiten implementar muchos de los principios pedagógicos de la Escuela Nueva y Moderna como son el aprendizaje a través de la experiencia y la actividad, la metodología de 
enseñanza por proyectos, la construcción de conocimiento por parte de los estudiantes, la colaboración, aprendizaje, personalización de la enseñanza, entre otros.

Finalmente hemos de indicar que una de las limitaciones de este trabajo es que no ha incorporado un análisis de las nuevas tecnologías emergentes que albergan grandes promesas para el cambio educativo como son las referidas a las aplicaciones de la inteligencia artificial en la enseñanza, el blockchain, las analíticas del big data, o la realidad virtual en la enseñanza entre otras. Asimismo, este artículo presenta las limitaciones propias de un ensayo académico. Por ello, como líneas de trabajo futuro recomendamos realizar revisiones bibliográficas más sistematizadas en torno a los cuatro tópicos que hemos identificado tanto en lo producido en lengua española con lo publicado en lengua inglesa con la finalidad de comprobar en qué medida los mismos están presentes en la actual agenda investigadora del campo de la Tecnología Educativa. Asimismo, también sería interesante realizar estudios de metaanálisis sobre investigaciones empíricas que hayan abordado estas temáticas en los últimos años.

\section{Referencias}

Adell, J. (2018). Más allá del instrumentalismo en tecnología educativa. En J. Gimeno (Ed.), Cambiar los contenidos, cambiar la educación (pp.116-128). Morata.

Adell, J. y Castañeda, L. (2012). Tecnologías emergentes, ¿pedagogías emergentes? En J. Hernández, M. Pennesi, D. Sobrino, A. Vázquez (Coords.), Tendencias emergentes en educación con TIC (pp. 13-33). Espiral.

Adell, J. y Castañeda, L. (2015). Las pedagogías escolares emergentes. Cuadernos de Pedagogía, 462, 21-25.

Alto Comisionado para la Lucha contra la Pobreza Infantil. (2020). Pobreza infantily desigualdad educativa en España. https://www.comisionadopobrezainfantil.gob.es/sites/default/files/Informe\%20ACPI-Educaci\%C3\%B3n.pdf

Asamblea General Naciones Unidas. (2016). Promoción, protección y disfrute de los derechos humanos en internet. https://digitallibrary.un.org/record/845728/files/A_HRC_32_L-20-ES.pdf

Area, M. (2011). Los efectos del modelo 1:1 en el cambio educativo en las escuelas. Evidencias y desafíos para las políticas iberoamericanas. Revista Iberoamericana de Educación, 56(1), 49-74. https://rieoei.org/RIE/article/view/510/955

Area, M. (2015). La integración educativa de las TIC en el sistema escolar de Canarias: Las visiones y prácticas de los agentes educativos. Informe final de evaluación TSP. Repositorio institucional de la Universidad de La Laguna.

Area, M. (2017). La metamorfosis digital del material didáctico tras el paréntesis Gutenberg. Revista Latinoamericana de Tecnología Educativa, 16(2), 13-28. https://doi.org/10.17398/1695-288X.16.2.13

Area, M. (2020).Escuel@digit@l.Los materiales didácticos en la red. Graó.

Area, M., Miño, R., Rivera, P. y Alonso, C. (2020). Investigación sobre tecnologías educativas: Más allá de los artefactos. En J. M. Sancho (Coord.), Caminos y derivas para otra investigación educativa y social (pp. 223-236). Octaedro.

Area, M., Santana, P. y Sanabria, A. L. (2020). La transformación digital de los centros escolares. Obstáculos y resistencias. Digital Education Review, 37, 15-31. https://doi.org/10.1344/der.2020.37.15-31

BECTA. (2004). A review of the research literature on barriers to the uptake of ICT by teachers. British Educational Communications and Technology Agency. http://dera.ioe.ac.uk/id/eprint/1603

Bond, M., Zawacki-Richter, O. y Nichols, M. (2018). Revisiting five decades of educational technology research: A content and authorship analysis of the British Journal of Educational Technology. British Journal of Educational Technology, 50(1), 11-52 https://doi.org/10.1111/bjet.12730

Burbules, N. y Callister, T. (2001). Educación: Riesgos y promesas de las nuevas tecnologías de la información. Granica.

Cabero, J., Duarte, A. y Barroso, J. (1998). La piedra angular para la incorporación de los medios audiovisuales, informáticos y nuevas tecnologías en los contextos educativos: La formación y el perfeccionamiento del profesorado. Edutec. Revista Electrónica de Tecnología Educativa, 8, 1-13. https://doi.org/10.21556/edutec.1998.8 
Cabero, J. (2016). ¿Qué debemos aprender de las pasadas investigaciones en tecnología educativa? RIITE. Revista Interuniversitaria de Investigación en Tecnología Educativa, 1, 23-33. http://doi.org/10.6018/riite/2016/256741

Cabrera, L., Pérez, C. N. y Santana, F. (2020). ¿Se incrementa la desigualdad de oportunidades educativas en la enseñanza primaria con el cierre escolar por el coronavirus? International Journal of Sociology of Education, 9(3), 27-52. http://doi.org/10.17583/rise.2020.5613

Cabrol, M. y Severin, E. (2010). TICs en educación. Una innovación disruptiva. IADB.

Castañeda, L., Esteve, F. y Adell, J. (2018). ¿Por qué hace falta repensar y definir la competencia docente para el mundo digital? RED Revista de Educación a Distancia, 56, art 6. https://www.um.es/ead/red/56/castaneda_et_al.pdf

Castañeda, L., Salinas. J. y Adell, J. (2020). Hacia una visión contemporánea de la tecnología educativa. Digital Education Review, 37, 240-268.

Cuban, L. (1986). Teachers and machines. Teachers College Press.

Cobo, C. (2019). Acepto las condiciones: Usos y abusos de las tecnologías digitales. Fundación Santillana.

Christensen, C. M., Horn, M. B. y Johnson, C. W. (2008). Disrupting class: How disruptive innovation will change the way the world learns. McGraw-Hill.

Departamento de Educación, Universidades e Investigación. (2008). Modelo de madurez tecnológica del centro educativo. Departamento de Educación, Universidades e Investigación del Gobierno Vasco.

Ertmer, P. (1999). Addressing first- and second-order barriers to change: Strategies for technology integration. Educational Technology Research and Development, 47(4), 47-61.

Escudero, J. M., Martínez, B. y Nieto, J. M. (2018). Las TIC en la formación continua del profesorado en el contexto español. Revista de Educación, 382, 57-80. http://doi.org/10.4438/1988-592X-RE-2018-382-392

Esteve, F., Castañeda, L. y Adell, J. (2018). Un modelo holístico de competencia docente para el mundo digital. Revista Interuniversitaria de Formación del Profesorado, 91, 105-116.

European Schoolnet. (2006). The ICT impact report: A review of studies of ICT impact on schools. European communities.

Fuentes, A., López, J. y Pozo, S. (2019). Análisis de la competencia digital docente: Factor clave en el desempeño de pedagogías activas con realidad aumentada. REICE. Revista Iberoamericana Sobre Calidad, Eficacia y Cambio en Educación, 17(2), 27-42. https://doi.org/10.15366/reice2019.17.2.002

García-Zabaleta, E., Sánchez-Cruzado, C., Campión, R. S. y Sánchez-Compaña, M. T. (2021). Competencia digital y necesidades formativas del profesorado de educación Infantil. Un estudio antes y después de la Covid-19. Edutec. Revista Electrónica de Tecnología Educativa, 76, 90-108. https://doi.org/10.21556/edutec.2021.76

Gros, B. (2000). El ordenador invisible. Hacia la apropiación del ordenador en la enseñanza. Gedisa-UOC.

Gros, B. (2016). Retos y tendencias sobre el futuro de la investigación acerca del aprendizaje con tecnologías digitales. RED: Revista de Educación a Distancia, 50, art 10. https://doi.org/10.6018/red/50/10

Gimeno, J. (2011). Grandeza y miseria del libro de texto. En J. Gimeno, R. Feito, P. Perrenaud y M. Clemente (Eds.), Diseño, desarrollo e innovación del curriculum (pp. 113-118). Morata.

Harris, J., Mishra, P. y Koehler, M. (2009). Teachers' technological pedagogical content knowledge and learning activity types: Curriculum-based technology integration refrained. Journal of Research on Technology in Education, 41(4), 393-416. https://doi.org/10.1080/15391523.2009.10782536

Hew, K. F. y Brush, T. (2007). Integrating technology into K-12 teaching and learning: Current knowledge gaps and recommendations for future research. Educational Technology Research and Development, 55, 223-252.

Hsu, Y., Hung, J. y Ching, Y. (2013). Trends of educational technology research: More than a decade of international research in six SSCI-indexed refereed journals. Educational Technology Research and Development, 61(4), 685-705. https://doi.org/10.1007/s11423-013-9290-9

INTEF. (2017). Marco común de la competencia digital docente. Instituto Nacional de Tecnologías Educativas y Formación del Profesorado. 
ISTE. (2017). ISTE standards for educators. A guide for teachers and other professionals. International Society for Technology in Education.

Kampylis, P. Punie, Y. y Devine, J. (2015). Promoting effective digital-age learning. A European framework for digitally-competent educational organisations. JRC Science Hub. https://doi.org/10.2791/54070

Lugo, M. T. y Kelly, V. (2011). La matriz TIC. Una herramienta para planificar las tecnologías de la información y comunicación en las instituciones educativas. IIPE-UNESCO.

Lores, B., Sancho, P. y García, M. R. (2019). La formación de la competencia digital en los docentes. Profesorado, 23(4), 20-34. https://doi.org/10.30827/profesorado.v23i4.11720

Magana, S. (2017). Disruptive classroom technologies: A framework for innovation in education. Corwin.

Marín, V. I., Zawacki-Richter, O., Pérez Garcías, A. y Salinas, J. (2017). Tendencias en el ámbito de la tecnología educativa en Iberoamérica: 20 años de la revista Edutec-e. Edutec. Revista Electrónica de Tecnología Educativa, 59, a357. https://doi.org/10.21556/edutec.2017.59.836

Martínez Bonafé, J. (2010). El currículum y el libro de texto: Una dialéctica siempre abierta. En J. Gimeno (Comp.), Saberes e incertidumbres sobre el curriculum (pp. 276-299). Morata.

Mishra, P. y Koehler, M. (2006). Technological pedagogical content knowledge: A framework for teacher knowledge. Teachers College Record, 108(6), 1017-1054.

Natividad, G., Spector, J. M. y Evangelopoulos, N. (2018). An analysis of two decades of educational technology publications. Springer. https://doi.org/10.1007/978-981-13-0137-7

Nichols, R. G. y Allen-Brown, V. (1996). Critical theory and educational technology. En D. Jonassen (Ed.), Handbook of research for educational communications and technology (pp. 226-252). MacMillan.

OECD y Harvard Graduate School of Education. (2020). A framework to guide an education response to the Covid-19 pandemic of 2020. https://globaled.gse.harvard.edu/files/geii/files/framework_guide_v2.pdf

Pelgrum, W. J. (2001). Obstacles to the integration of ICT in education: Results from a worldwide educational assessment. Computers and Education, 37, 163-178. https://doi.org/10.1016/S0360-1315(01)00045-8

Pérez Gómez, A. (2012). Educarse en la era digital. Morata.

Postman, N. (1994). Tecnópolis. La rendición de la cultura a la tecnología. Círculo de Lectores.

Redecker C. y Punie Y. (2017). Digital competence framework for educators (DigCompEdu). JRC Science Hub.

Reimers, F. y Schleicher, A. (2020a). Schooling disrupted, schooling rethought. How the Covid-19 pandemic is changing education. OCDE.

Reimers, F. y Schleicher, A. (2020b). A framework to guide an education response to the Covid-19 pandemic of 2020. OECD.

Sancho, J. M., Bosco, A., Alonso, C. y Sánchez, J. A. (2015). Formación del profesorado en tecnología educativa. De cómo las realidades generan los mitos. RELATEC Revista Latinoamericana de Tecnología Educativa, 14(1), 17-34. https://doi.org/10.17398/1695-288X.14.1.17

Selwyn, N. (2011). Schools and schooling in the digital age. A critical analysis. Routledge.

Selwyn, N. (2014). Distrusting educational technology critical. Questions for changing times. Routledge.

Severin, E. (2011). Tecnologías para la educación. Un marco para la acción. Banco Interamericano de Desarrollo.

Severin, E., Peirano, C. y Falck, D. (2012). Guía básica para la evaluación de proyectos. Tecnologías para la educación. Banco Interamericano de Desarrollo.

UNESCO. (2008a). ICT competency standards for teachers. UNESCO.

UNESCO. (2018b) UNESCO ICT competency standards for teachers. UNESCO.

UNESCO. (2020a). Global monitoring of school closures caused by COVID-19. UNESCO.

UNESCO. (2020b). National education responses to COVID-19. Summary report of UNESCO s'online survey. UNESCO. 
UNESCO. (2020c). Framework for reopening schools. UNESCO.

Valiente, O. (2011). Los modelos 1:1 en educación. Prácticas internacionales, evidencia comparada e implicaciones políticas. Revista Iberoamericana de Educación, 56(1), 113-134.

Valverde, J. (2015). El proyecto de educación digital en un centro educativo. Síntesis.

West, R. E. y Borup, J. (2014). An analysis of a decade of research in 10 instructional design and technology journals. British Journal of Educational Technology, 45(4), 545-556.

Willianson, B. y Hogan, A. (2020). La comercialización y la privatización en y de la educación en el contexto de la Covid-19. https://ei-ie-al.org/sites/default/files/docs/20200708_102224_la_comercializacion_y_la_privatizacion_en_y_ de_la_educacion_en_el_contexto_de_la_covid-19.pdf

Yanes, J. y Area, M. (1998). El final de las certezas. La formación del profesorado ante la cultura digital. Pixel-Bit. Revista de Medios y Educación, 10, 25-36.

Vuorikari, R., Punie, Y., Carretero, S. y Van den Brande. L (2016). DigComp 2.0: The digital competence framework for citizens. Update phase 1: The conceptual reference model. JRC Science Hub.

\section{Breve CV de los autores}

\section{Manuel Area}

Doctor en Pedagogía y Catedrático de Didáctica y Organización Escolar del Departamento de Didáctica e Investigación Educativa de la Universidad de La Laguna. Es investigador principal y director del grupo Laboratorio de Educación y Nuevas Tecnologías. EDULLAB. Su ámbito de docencia e investigación es la Tecnología Educativa en el que ha realizado más de doscientas publicaciones (índice h: 57, índice i10: 178), dirigido varios proyectos de investigación $\mathrm{I}+\mathrm{D}$ tanto de ámbito autonómico, nacional y europeo. Fue fundador y presidente de la Red Universitaria de Tecnología Educativa (RUTE). Colabora regularmente con diversas universidades latinoamericanas. Email: manarea@ull.edu.es

ORCID ID: https://orcid.org/0000-0003-0358-7663

\section{Jordi Adell}

Doctor en Ciencias de la Educación y Licenciado en Filosofía y Ciencias de la Educación por la Universidad de Valencia. Actualmente es profesor titular de universidad del área de Didáctica y Organización Escolar en el Dpto. de Pedagogía de la Universitat Jaume I de Castellón. Su actividad docente está relacionada desde hace muchos años con las nuevas tecnologías de la información y la comunicación aplicadas a la educación. Es coordinador del Grupo de Investigación sobre Enseñanza, Aprendizaje y Tecnología (GREAT). También dirige el Centro de Educación y Nuevas Tecnologías (CENT) de la misma universidad, unidad dedicada a la mejora de la calidad de la enseñanza y el aprendizaje universitarios mediante el uso de las tecnologías digitales de la información y la comunicación. Email: jordi@edu.uji.es

ORCID ID: http://orcid.org/0000-0003-4369-6485 